\title{
26 Research Soure \\ Fisetin Induced Cell Death in Human Ovarian Cancer Cell Lines via ZBP1 Mediated Necroptosis.
}

\section{Yaxian Liu}

Tianjin Medical University Baodi Clinical College: Tianjin Baodi Hospital

\section{Wenhong Cao}

Tianjin Medical University Baodi Clinical College: Tianjin Baodi Hospital

\section{Yanhui Zhao}

Tianjin Medical University Baodi Clinical College: Tianjin Baodi Hospital

\section{Lijuan Shan}

Tianjin Medical University Baodi Clinical College: Tianjin Baodi Hospital

Shuhai Lan ( $\sim$ lanshuhuatj@outlook.com )

Tianjin Medical University Baodi Clinical College: Tianjin Baodi Hospital https://orcid.org/0000-00020586-9616

\section{Research Article}

Keywords: gynecological malignancy, cancer, fisetin, necroptosis

Posted Date: June 1st, 2021

DOl: https://doi.org/10.21203/rs.3.rs-520727/v1

License: (c) (1) This work is licensed under a Creative Commons Attribution 4.0 International License.

Read Full License 


\section{Abstract}

Background: Ovarian cancer leads to severe female mortality among all reproductive cancers. Fisetin, a natural flavonoid, exerts pharmacological characteristics on inhibiting cancer growth from various origins. Although multiple mechanisms involving in regulating cell death, there is still unclear if and how fisetin exhibits anti-cancer effect on ovarian cancer. The presented study aimed to evaluate cell apoptotic and necroptotic processes occurring in ovarian carcinoma $(\mathrm{OC})$ cell lines induced by fisetin

Methods: Cell growth was evaluated by MTT assay in both OC cell lines treated with or without fisetin. Annexin V/Propidium iodide staining followed by flow cytometry were used to characterize fisetin induced cell death. The apoptotic process was suppressed by z-VAD intervention then cell necroptosis was assessed by introducing ZBP1 knockdown OC cell lines coupled with fisetin intervention. The expression of necroptosis-related mediators and migration capability of respective cells were evaluated by western blotting and in vitro cell invasion assay.

Result: Fisetin successfully reduced cell growth on both OC cell lines in a dose-dependent manner. Both apoptosis and necroptosis were induced by fisetin. Suppression on cell apoptotic process failed to enhance proliferation of fisetin treated cells. The induced cell death as well as robust expression of necroptotic markers RIP3 and MLKL were alleviated by knocking down the expression of ZBP1 protein in both $\mathrm{OC}$ cell lines.

Conclusion: The present study demonstrated in vitro evidence supporting that both apoptosis and necroptosis were involved in fisetin induced OC cell death, while ZBP1 regulates necroptotic process via RIP3/MLKL pathway.

\section{Introduction}

Ovarian cancer $(\mathrm{OC})$ ranks as the second most common gynecological malignancy, which is also one of the leading causes of cancer-related mortality in women[1]. Although multiple types of chemotherapies and surgeries have been applied in OC treatment, the prognosis and long-term survival rate of OC patients are still poor[2, 3]. Due to its high heterogeneity and complex cellular origins, it still challenges the researchers and clinicians to screen substances and target critical factors which are able to effectively promote cell death or control the ovarian carcinogenesis[4]. However, OC cells depend on both inherent and acquired mechanisms to resist the effect of chemotherapeutics and escape cell death[5]. Therefore, to understand the process and mechanisms involved in $\mathrm{OC}$ cell death under chemotherapies have become important for optimizing current treatment outcome of ovarian cancer.

Fisetin $\left(7,3^{\prime}, 4^{\prime}\right.$-flavon-3-ol) is a flavonoid substance which can be extracted from a variety of plants, such as cucumbers, onions, apples or strawberries[6]. Fisetin has been reported to possess multiple biological processes with therapeutic effects, including antioxidant, anti-inflammatory, and prevention or inhibition to cancer development [7-10]. As for its anticancer effects, many types of malignant cancer cells were involved. Fisetin arrested cell cycle in colon cancer cell by inhibiting activities of cyclin-dependent 
kinases[11]. In osteosarcoma, fisetin promote cell death via mitochondrial apoptosis, arresting cell cycle and inhibition of cell migration[12] or via mediating multiple signaling pathways[13]. Jia etc. found that fisetin reduced proliferation of pancreatic cancer cells by inducing autophagy via endoplasmic reticulum and mitochondrial related pathways[14].

Similar to other solid malignant cancer, various types of cell death processes have been discovered in OC over the past decade. Apoptosis occurs via two main mechanisms: a receptor-dependent extrinsic pathway and a mitochondria-dependent intrinsic pathway[15]. Both pathways have been reported in leading cell death of ovarian cancer[16-18]. However, ovarian cancer cells are also able to escape therapeutic-induced apoptosis via counterbalancing the effectors and antagonists regulating caspase related pathways $[19,20]$. Necroptosis serves as an alternative approach of programmed cell death in a caspase-independent pathway and participates the regulation towards cancer metastasis, tumor progression or cancer immunity regulation, which may be another significant mechanism regarding to optimizing ovarian cancer treatment outcome[21,22]. Besides, ferroptosis and tumor microenvironment also participate in initiating OC cell death[23, 24].

In this study we intend to focus on the necroptosis of $\mathrm{OC}$ with fisetin intervention. Recent studies have identified the ZBP1 as a potential mediator in the regulation of cell death, including various types of cancer[25]. However, there is still lack of evidence about how ZBP1 regulate cell death in ovarian cancer. To get insight into the mechanism of fisetin in the treatment of ovarian cancer, in this study, we firstly proved the cell death of different OC cell lines, A2780 and VOCAR-3, can be initiated by fisetin in a dosedependent manner. Then both cell apoptosis and necroptosis were involved in cell death induced by fisetin. After inhibiting apoptotic approach, we observed ZBP1 played a significant role in mediating fisetin induced cell necroptosis in OC cell lines via ZBP1 mediated RIP3/MLKL necroptotic pathway.

\section{Materials And Methods}

\section{Reagents and antibodies}

Fisetin and z-VAD were purchased from Sigma, USA. The primary antibodies used in this study for western blotting were as follows: anti-HMGB1(Invitrogen, NY, USA), 1:1000 dilution; anti-ZBP1 (R\&D system, USA), 1:1000 dilution; anti-RIP3(Calbiochem, San Diego, CA), 1:1000 dilution; anti-

RIP1(Calbiochem, San Diego, CA), 1:1000 dilution; anti-MLKL (BD Biosciences, USA), 1:1000 dilution; antiGAPDH (Santa Cruz, CA, USA), 1:5000 dilution.

\section{Cell culture}

Human ovarian carcinoma (OC) cell lines was obtained from Procell (Procell, China) and cultured in DMEM medium (Hyclone, USA) in which $10 \%$ fetal bovine serum (Gibico, USA) and $1 \%$ penicillinstreptomycin (Sigma, USA) were supplemented. Both cell lines were cultured in a $37 \%$ with $5 \% \mathrm{CO}_{2}$ atmosphere. Fisetin was dissolved in dimethyl sulfoxide (DMSO) to a series concentration of $0,25,50$ or $100 \mu \mathrm{mol} / \mathrm{L}$ then added into the culture medium to treat both cell lines for $72 \mathrm{hr}$. v-ZAD (dissolved into 30 
$\mu \mathrm{mol} / \mathrm{L}$ concentration) was added into $100 \mu \mathrm{mol} / \mathrm{L}$ fisetin treated cells for $72 \mathrm{hr}$ to inhibit the apoptotic process.

\section{Cell Viability Assay}

The effect of fisetin on cell viability was examined by the 3-(4,5- dimethylthiazol-2-yl)-2,5-diphenyl-2Htetrazolium bromide (MTT) assay. Briefly, A2780 and VOCAR-3 cells in both treated and control group were seeded in 96 -well plates at the concentration of $75 \mu \mathrm{mol} / \mathrm{L}$ cells per well. Cells were then incubated for $12 \mathrm{~h}$ following fisetin treatment with a series of concentrations as mentioned. After incubation for $72 \mathrm{~h}$, MTT was added into each well following $4 \mathrm{~h}$ incubation. The medium was then washed for 3 times to remove MTT. After stabilizing the blue formazan crystals by adding $150 \mu \mathrm{L}$ of DMSO, the absorbance at $570 \mathrm{~nm}$ was detected with a microplate reader (Bio-Tek Instruments, VT) to determine the concentration of blue crystals.

\section{Cell death analysis}

Annexin V-FITC apoptosis detection kit (Sigma-Aldrich) was used for the detection of apoptotic cells. Cells were double-stained with $\mathrm{PI}$ and Annexin- $\mathrm{V}$ with a binding buffer for $30 \mathrm{~min}$ at room temperature (RT). Apoptotic and necroptotic cells were analyzed with flow cytometry.

\section{Transfection of ZBP1 siRNA}

ZBP1 siRNAs (Santa Cruz Biotechnology, Santa Cruz, CA) were introduced to block the endogenous ZBP1 gene expression. Briefly, the ZBP1 siRNA (Thermal fisher, USA) was transfected into A2780 cells using siRNA transfection kit (Santa Cruz Biotechnology, Santa Cruz, CA) and following manufacturer's instructions. After $24 \mathrm{hr}$ transfection, fisetin + Z-VAD treatment was then followed. The levels of ZBP1 protein in both cell lines were analyzed by Western blotting.

\section{In vitro invasion assay}

An in vitro cell invasion assay was introduced to measure the migration ability of both ZBP knockdown (ZBP1-/-) and control cells after fisetin + Z-VAD treatment using the Matrigel-coated Transwell chamber (Corning, MA, United States). Briefly, both types of cells at a density of 24 cells/well were seeded onto the upper chambers in a serum-free medium with fisetin + z-VAD, while DMEM medium containing $10 \%$ FBS and $1 \%$ penicillin-streptomycin was filled into the lower chamber. After incubation for $24 \mathrm{~h}$, the cells on top surface were removed while cells on the lower surface of the membranes were fixed with $4 \%$ paraformaldehyde for $10 \mathrm{~min}$ and stained using $0.25 \%$ crystal violet for $15 \mathrm{~min}$. The invaded cells were introduced to an MTT assay.

\section{Cell mitochondria isolation}

Cell mitochondria isolation was performed using a Cell Mitochondria Isolation Kit (Thermal fisher, USA) according to the manufacturer's instructions. Briefly, the cells were washed with PBS and digested with trypsin, then the cells were centrifuged at a speed of $100-200 \mathrm{~g}$ for $5 \mathrm{~min}$ to collect alkalotic cells. We then transferred the cells to clean tubes and homogenized the cells 10-30 times. The cell homogenate 
was centrifuged at a speed of $600 \mathrm{~g}$ at $4^{\circ} \mathrm{C}$ for $10 \mathrm{~min}$. We transferred the supernatant to another clean tube and centrifuged it at a speed of $11000 \mathrm{~g}$ at $4^{\circ} \mathrm{C}$ for $10 \mathrm{~min}$. The supernatant was cytosol, and the deposit was mitochondria.

\section{Real-time PCR}

RNA was extracted from isolated mitochondria by the PureLink RNA mini kit (Thermo Fisher Scientific, USA). The quantity and quality of extracted RNA were analyzed by using NanoDrop 2000

Spectrophotometer. Extracted RNA was prepared to synthesize cDNA according to the Quantinova reverse transcription kit. The Quantinova SYBR Green PCR Kit was used to determine mRNA expression on an Applied Biosystems PCR machine $\left(95^{\circ} \mathrm{C}\right.$ for $10 \mathrm{~min}, 40$ cycles of $95^{\circ} \mathrm{C}$ for $15 \mathrm{~s}$ and $60^{\circ} \mathrm{C}$ for $30 \mathrm{~s}$, and final extension step of $60^{\circ} \mathrm{C}$ for $30 \mathrm{~s}$ ). Sequence of the primers used in the qRT-PCR experiment was listed as followed:

Cyt-c forward primer: 5'-CTG GGTGACGAGTGAAACTG-3'; reverse primer: 5'-TGAGCACAACAGGAACTGGA3 ; primer length in bp: 104bp.

GAPDH forward primer: 5'-GAAATCCCATCACCATCTTCCAGG-3'; reverse primer: 5'GAGCCCCAGCCTTCTCCATG-3'; primer length in bp: 120bp.

\section{Western blot analysis}

Cells were cultured in absence or presence of respective intervention, then harvested and lysed in RIPA buffer for collecting the total protein. After iced incubation for $30 \mathrm{~min}$, lysates were centrifugated at $12000 \mathrm{~g}$ for $5 \mathrm{~min}$ to separate the supernatants. The concentration of protein containing in the supernatants were measured by the BCA kit according to the manufacturer's instructions. Next, $30 \mu \mathrm{g}$ denatured protein samples were separated on SDS-PAGE gels and then transferred to PVDF membranes (Bio-Rad). After incubation of the membranes with primary antibodies at $4^{\circ} \mathrm{C}$ overnight, the samples were incubated with the secondary antibodies conjugated with horseradish peroxidase for $1 \mathrm{~h}$ at room temperature. The blots were visualized using a Leica Imaging System.

\section{Statistical Analysis}

The statistical analysis was completed with the software package SPSS 23.0(SPSS Inc.; Chicago, IL, USA) and GraphPad Prism 6 (San Diego, CA) software. Data were presented as mean \pm standard deviation. The Student's t-test was used to compare the means of groups and P-values $\leq 0.05$ were considered significant.

\section{Results}

\section{Fisetin suppressed cell proliferation in human ovarian cancer cell lines in vitro}


In order to assess the cytotoxicity of fisetin (the chemical structure in Fig. 1A) to OC cells. A2780 and VOCAR-3 cell lines were treated by fisetin in different concentrations $(0,25,50$ or $100 \mu \mathrm{mol} / \mathrm{L})$ for 72 hours to determine the cell viability. The MTT assay indicated the significant inhibition to cell proliferative activities in both cell lines after fisetin treatment in all concentrations (Fig. 1B). Only about $20 \%$ cells of both cell lines survived after $100 \mu \mathrm{mol} / \mathrm{L}$ fisetin. (Fig. 1C).

\section{Fisetin induces not only apoptosis in OC cell lines}

The decrease in number of vital cells may attributed to robust cell death. Therefore, we verified this hypothesis by using Annexin V/Propidium lodiden (AV/PI) staining followed by flow cytometry analysis. Fisetin mediated A2780 cells with lower percentage in vital cells, indicated by $\mathrm{AV}(-) / \mathrm{PI}(-)$ (Fig. 2A). With the intervention by $z-V A D$, a pan caspase inhibitor, the fisetin induced cell death were significantly reduced compared to those without $z-V A D$ treatment (Fig. 2B).

Due to the release of Cyt-c during apoptosis from mitochondria, the translation level of cyt-c mitochondrial RNA was significantly lower in fisetin treated cells than untreated ones in the present study, indicating more intensive cell apoptosis was induced by fisetin (Fig. 2C). By inhibiting apoptotic process using z-VAD, the percentage of apoptotic A2780 cells following fisetin treatment significantly decreased compared to cells without z-VAD antagonism. However, compare to non-treated control group, the suppression on A2780 cell apoptosis were not able to significantly enhance cell growth at different time points following fisetin treatment (Fig. 2D). Similar trends were also observed in VOCAR-3 cell line (Fig. 2E-H). Consequently, the death induced by fisetin in OC cell lines does not simply attribute to apoptosis.

\section{Fisetin induced cell necroptosis were mediated by ZBP1 involved RIP3/MLKL pathway}

The expression of necroptotic markers was analyzed through Western Blotting. A2780 with fisetin + z-VAD treatment showed a significant increase in the protein levels of ZBP1, RIP3, and MLKL compared to control, whereas the level of HMGB1, an inflammation related marker, showed no significant difference between both groups (Fig. 3A-B). As for VOCAR-3 cell line, similar changes occurred between group in which fisetin and $z-V A D$ were involved compared to non-treated control group (Fig. $3 C-D$ ). These results indicated the necroptosis induced by fisetin in both OC cell lines were mediated by ZBP1/RIP3/MLKL pathway.

\section{ZBP1 knockdown compensated the effects by fisetin + z- VAD intervention and resumed the viability of $O C$ cell lines}

To gain insights of the mechanism, how the fisetin induced OC cell necroptosis was regulated. Due to the possible dominant roles of ZBP1/RIP3/MLKL pathway in controlling necroptotic process, we hypothesized that ZBP1 plays central role in mediating OC cell necroptosis following fisetin treatment. In 
order to eliminate the effects from ZBP1 protein, A2780 cells were firstly transfected with siRNA interfering ZBP1 expression. By western blotting ZBP1 level in either transfected or control cells, less ZBP1 protein was synthesized in ZBP1-/- cells than in control ones (Fig. 4A-B). Using these transfected cells into an in vitro invasion assay, we observed that the knockdown of ZBP1 blocked necroptotic process on A2780 cells (Fig. 4C), evidenced by more robust migration activity on ZBP1-/-cells following fisetin + z-VAD treatment (Fig. 4D), whereas the existence of ZBP1 significantly inhibited the cell migration in control group (Fig. 4C, D). Next we conjectured that a decline in endogenous ZBP1 level might also impact the cell necroptosis by downregulate the downstream markers, RIP3 and MLKL. The western blot on both markers showed significant inhibition in expression after ZBP1 was eliminated compared to that of control group in which OC cell necroptosis was induced by fisetin + Z-VAD treatment (Fig. 4E). The relative expression level of downstream RIP3 was nearly 4 times lower than non-transfected control (Fig. 4F). Similar inhibition was also observed in MLKL expression in fisetin + v-ZAD induced cellular necroptosis (Fig. 4E-F).

As for another representative cell line mimicking OC, ZBP1 siRNA was also successfully blocked the targeted gene expression in OVCAR-3 cells (Fig. 5A, B), significantly compensating the decreased cell migration and cell death (Fig. 5C, D). RIP3 and MLKL expression level shared similar suppressed trends in OVCAR-3 cells with or without transfection (Fig. 5E, F), which were similar to those of conditions on A2780 cell line. Consequently, we verified the initial hypothesis ZBP1 acted as a central regulator in governing cell necroptosis in both A2780 and OVCAR-3 cell lines.

\section{Discussion}

The past decades have witnessed that natural products have become the new territory in therapeutic development. Fisetin, a natural flavonoid, exerts pharmacological characteristics in mediating inflammation, inhibiting cancer, antioxidant and promoting angiogenesis[7-9]. It has been demonstrated as a phytochemical to exhibit cytotoxicity and inhibit proliferation in various types of cancer, including osteosarcoma[26], nonsmall cell lung carcinoma[27] and breast cancer[28]. These anticancer effects were also mediated by multiple signaling pathways related to cell death regulation. However, it still needs systemic research characterizing the properties and mechanism of fisetin in inhibiting ovarian cancer growth. Therefore, the present study provides in vitro evidence demonstrating the effects of fisetin in promoting cell death of various ovarian cancer cell lines via cell apoptosis and necroptosis. ZBP1 plays as a key factor in mediating cell necroptotic process induced by fisetin in OC cell lines.

To begin with, we introduced two types of cell lines representing human OC cells and verified the cytotoxicity of fisetin in different concentration by MTT assay. The results indicated a dose-dependent manner of fisetin in inhibiting proliferation of both cell lines. Among all concentrations selected, 100 $\mu \mathrm{mol} / \mathrm{L}$ showed the strongest inhibitory effects in our study. This dose-dependent manner of fisetin was verified in a similar study targeting on the other type of OC cell line, namely the SKOV3 cell, in which up to $300 \mu \mathrm{mol} / \mathrm{L}$ of fisetin were involved[29]. The results of MTT assay demonstrated greater inhibition on cell viability appeared in $100 \mu \mathrm{mol} / \mathrm{L}$ group than that in $30 \mu \mathrm{mol} / \mathrm{L}$. Although shown more robust growth 
inhibitory effect in $300 \mu \mathrm{mol} / \mathrm{L}$ group, the difference of cell viability between 100 and $300 \mu \mathrm{mol} / \mathrm{L}$ was relatively less compared to that between 30 and $100 \mu \mathrm{mol} / \mathrm{L}$ group. Thus, $100 \mu \mathrm{mol} / \mathrm{L}$ of fisetin were able to induce strong cell death in $\mathrm{OC}$ cell lines for further experiments.

Cell apoptosis is a common process involved in chemical induced cell death among various cancers[30, 31]. Studies also reported that fisetin induced cell apoptosis in different types of malignant tumor cell lines. Here, we firstly verified this hypothesis by establishing Annexin V (AV) and Propidium lodide (PI) double staining followed by flow cytometry analysis[32]. Higher percentage of $\mathrm{AV}(+) / \mathrm{PI}(+)$ and $\mathrm{AV}(-) / \mathrm{PI}(+)$ indicated apoptosis at different stages were induced by fisetin, causing less expression of Cyt-c in fisetin treated cells. Z-VAD acts as an effective pan-caspase inhibitor which could significantly block the mitochondria-driven intrinsic apoptotic process. However, compared to cells only with fisetin treatment, zVAD administration were unable to reverse the inhibitory effects by fisetin on cell proliferation. These results indicated the possibility that other mechanisms rather than cell apoptosis might contribute to cell death induced by fisetin in the selected $\mathrm{OC}$ cell lines.

z-VAD were reported to enhance cell necroptotic process while blocking cell apoptosis. Meanwhile, the present cytometric results also indicated the possibility that cell necroptosis occurred, evidenced by strong signal of $\mathrm{AV}(+) / \mathrm{PI}(+)$. Thus, we hypothesized that cell necroptotic process and relative signaling pathway regulates fisetin induced $\mathrm{OC}$ cell death.

Z-DNA-binding protein 1 (ZBP1) functions as a central regulator of cell necroptosis in diverse conditions [25, 33, 34]. ZBP1 facilitated RIPK3 activation and its binding to MLKL[34, 35]. The activation of RIP3 is the key to activate MLKL mediated cell necroptosis. In the present study, western blotting targeting the expression level of ZBP1, RIP3 and MLKL were measured between both OC cell lines with or without fisetin $+z-V A D$ treatment. The results supported the hypothesis that cell necroptosis attributed to cell death induced by fisetin. To validate the initiative role of ZBP1 in mediating the necroptosis, we successfully established ZBP1 siRNA transfected A2780 and OVCAR-3 cells to knockdown the ZBP1 expression. Then the invasion assay demonstrated stronger migration was observed in ZBP1 knockdown cells after fisetin $+z-V A D$ treatment. The downstream mediators RIP3 and MLKL were also be downregulated without ZBP1 (Fig. 6).

\section{Conclusions}

To the best of our knowledge, the presented data in this study demonstrated in vitro evidence indicating both cell apoptosis and necroptosis play important roles in the process of fisetin-initiated OC cell death. ZBP1 mediated fisetin induced cell necroptosis in OC cell lines via RIP3/MLKL pathway. However, the contribution of each mentioned mechanism in cell death process by fisetin need to be further quantified.

\section{Declarations}

\section{Ethics approval and consent to participate}


Not applicable.

\section{Consent for publication}

Not applicable.

\section{Availability of data and materials}

The dataset supporting the conclusions of this article is included within the article and its additional files.

\section{Competing interests}

The authors declare that they have no competing interests.

\section{Funding}

No funding was received.

\section{Authors' contributions}

Yaxian Liu, Shuhai Lan supervised the study and finalized the manuscript. Yaxian Liu, Wenhong Cao, Yanhui Zhao, Lihuan Shan engaged in this study design, performed bioinformatic analysis and drafted the manuscript.

\section{Acknowledgements}

Not applicable.

\section{References}

1. Torre, L.A., et al., Ovarian cancer statistics, 2018. CA Cancer J Clin, 2018. 68(4): p. 284-296.

2. Kurosaki, A., et al., Serum folate receptor alpha as a biomarker for ovarian cancer: Implications for diagnosis, prognosis and predicting its local tumor expression. Int J Cancer, 2016. 138(8): p. 19942002.

3. Chiang, Y.C., et al., Trends in incidence and survival outcome of epithelial ovarian cancer: 30-year national population-based registry in Taiwan. J Gynecol Oncol, 2013. 24(4): p. 342-51.

4. Kroeger, P.T., Jr. and R. Drapkin, Pathogenesis and heterogeneity of ovarian cancer. Curr Opin Obstet Gynecol, 2017. 29(1): p. 26-34.

5. Al-Alem, L.F., et al., Understanding and Targeting Apoptotic Pathways in Ovarian Cancer. Cancers (Basel), 2019. 11(11).

6. Arai, Y., et al., Dietary intakes of flavonols, flavones and isoflavones by Japanese women and the inverse correlation between quercetin intake and plasma LDL cholesterol concentration. J Nutr, 2000. 130(9): p. 2243-50. 
7. Kashyap, D., et al., Fisetin: A bioactive phytochemical with potential for cancer prevention and pharmacotherapy. Life Sci, 2018. 194: p. 75-87.

8. Pal, H.C., R.L. Pearlman, and F. Afaq, Fisetin and Its Role in Chronic Diseases. Adv Exp Med Biol, 2016. 928: p. 213-244.

9. Rengarajan, T. and N.S. Yaacob, The flavonoid fisetin as an anticancer agent targeting the growth signaling pathways. Eur J Pharmacol, 2016. 789: p. 8-16.

10. Zhang, H.F., et al., Fisetin alleviates sepsis-induced multiple organ dysfunction in mice via inhibiting p38 MAPK/MK2 signaling. Acta Pharmacol Sin, 2020. 41(10): p. 1348-1356.

11. Lu, X., et al., Fisetin inhibits the activities of cyclin-dependent kinases leading to cell cycle arrest in HT-29 human colon cancer cells. J Nutr, 2005. 135(12): p. 2884-90.

12. Xing, C., et al., Anticancer activity of Fisetin against the human osteosarcoma cell lines involves G2/M cell cycle arrest, mitochondrial apoptosis and inhibition of cell migration and invasion. J BUON, 2020. 25(2): p. 1022-1027.

13. Fu, C.Y., et al., Fisetin activates Hippo pathway and JNK/ERK/AP-1 signaling to inhibit proliferation and induce apoptosis of human osteosarcoma cells via ZAK overexpression. Environ Toxicol, 2019. 34(8): p. 902-911.

14. Jia, S., et al., Fisetin induces autophagy in pancreatic cancer cells via endoplasmic reticulum stressand mitochondrial stress-dependent pathways. Cell Death Dis, 2019. 10(2): p. 142.

15. Fulda, S. and K.M. Debatin, Extrinsic versus intrinsic apoptosis pathways in anticancer chemotherapy. Oncogene, 2006. 25(34): p. 4798-811.

16. Lee, J.Y., et al., Therapeutic potential of alpha,beta-thujone through metabolic reprogramming and caspase-dependent apoptosis in ovarian cancer cells. J Cell Physiol, 2020.

17. Kostova, l., et al., The role of caspase-8 in the tumor microenvironment of ovarian cancer. Cancer Metastasis Rev, 2020.

18. Binju, M., et al., Therapeutic Inducers of Apoptosis in Ovarian Cancer. Cancers (Basel), 2019. 11(11).

19. Sasaki, H., et al., Down-regulation of X-linked inhibitor of apoptosis protein induces apoptosis in chemoresistant human ovarian cancer cells. Cancer Res, 2000. 60(20): p. 5659-66.

20. Yang, X., et al., Regulation of HtrA2/Omi by X-linked inhibitor of apoptosis protein in chemoresistance in human ovarian cancer cells. Gynecol Oncol, 2005. 97(2): p. 413-21.

21. Gong, Y., et al., The role of necroptosis in cancer biology and therapy. Mol Cancer, 2019. 18(1): p. 100.

22. McCabe, K.E., et al., Triggering necroptosis in cisplatin and IAP antagonist-resistant ovarian carcinoma. Cell Death Dis, 2014. 5: p. e1496.

23. Yang, Y., et al., Tumor Microenvironment in Ovarian Cancer: Function and Therapeutic Strategy. Front Cell Dev Biol, 2020. 8: p. 758.

24. Xia, X., et al., The Relationship between Ferroptosis and Tumors: A Novel Landscape for Therapeutic Approach. Curr Gene Ther, 2019. 19(2): p. 117-124. 
25. Kuriakose, T. and T.D. Kanneganti, ZBP1: Innate Sensor Regulating Cell Death and Inflammation. Trends Immunol, 2018. 39(2): p. 123-134.

26. Chen, J.K., et al., Fistein Suppresses Human Osteosarcoma U-2 OS Cell Migration and Invasion via Affecting FAK, uPA and NF-kB Signaling Pathway In Vitro. In Vivo, 2019. 33(3): p. 801-810.

27. Tabasum, S. and R.P. Singh, Fisetin suppresses migration, invasion and stem-cell-like phenotype of human non-small cell lung carcinoma cells via attenuation of epithelial to mesenchymal transition. Chem Biol Interact, 2019. 303: p. 14-21.

28. Guo, G., et al., Fisetin induces apoptosis in breast cancer MDA-MB-453 cells through degradation of HER2/neu and via the PI3K/Akt pathway. J Biochem Mol Toxicol, 2019. 33(4): p. e22268.

29. Xiao, X., et al., Fisetin and polymeric micelles encapsulating fisetin exhibit potent cytotoxic effects towards ovarian cancer cells. BMC Complement Altern Med, 2018. 18(1): p. 91.

30. Sundarraj, K., et al., Fisetin, a phytopolyphenol, targets apoptotic and necroptotic cell death in HepG2 cells. Biofactors, 2020. 46(1): p. 118-135.

31. Carneiro, B.A. and W.S. El-Deiry, Targeting apoptosis in cancer therapy. Nat Rev Clin Oncol, 2020. 17(7): p. 395-417.

32. Crowley, L.C., et al., Quantitation of Apoptosis and Necrosis by Annexin V Binding, Propidium lodide Uptake, and Flow Cytometry. Cold Spring Harb Protoc, 2016. 2016(11).

33. Devos, M., et al., Sensing of endogenous nucleic acids by ZBP1 induces keratinocyte necroptosis and skin inflammation. J Exp Med, 2020. 217(7).

34. Jiao, H., et al., Z-nucleic-acid sensing triggers ZBP1-dependent necroptosis and inflammation. Nature, 2020. 580(7803): p. 391-395.

35. Tang, D., et al., The molecular machinery of regulated cell death. Cell Res, 2019. 29(5): p. 347-364.

\section{Figures}

A

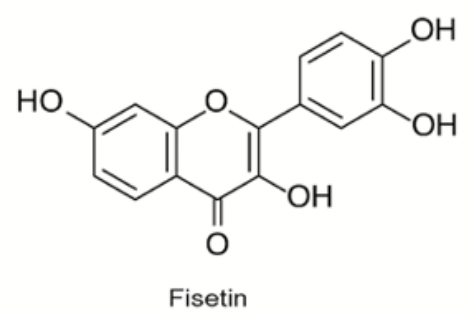

B

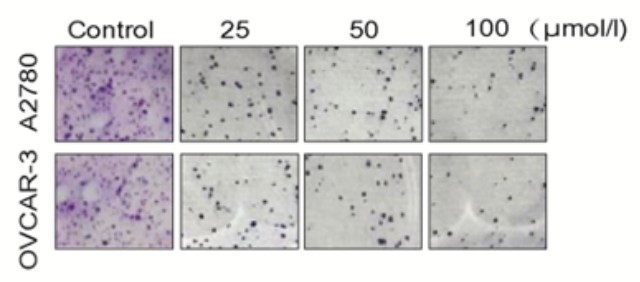

C

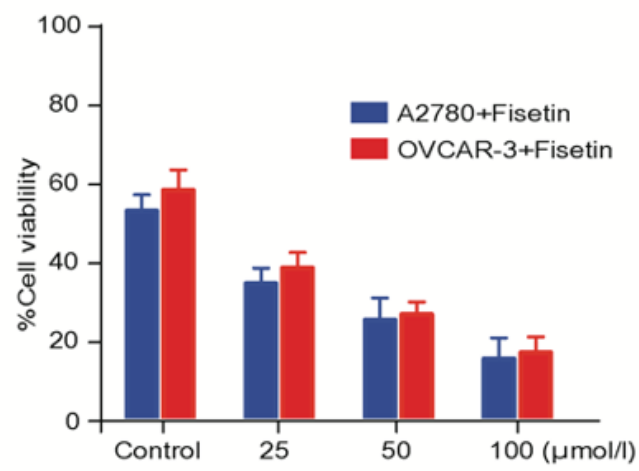

\section{Figure 1}

Fisetin inhibited the proliferation of both human ovary carcinoma cell lines (Ho8910 and A2780) in a dose-dependent manner. (A) The chemical structure of fisetin. (B) The MTT assay indicated the reduced 
proliferation of both $\mathrm{OC}$ cell lines treated after fisetin on incremental concentration $(25,50$ and 100 $\mathrm{mmol} / \mathrm{L}$ ) for $72 \mathrm{~h}$. (C) Quantification of vital cells after fisetin treatment.
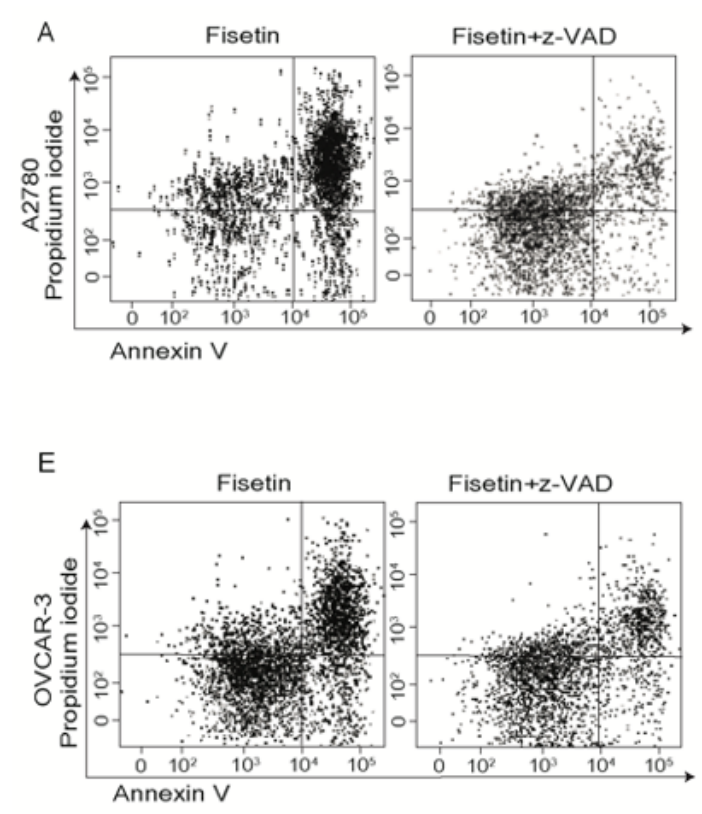
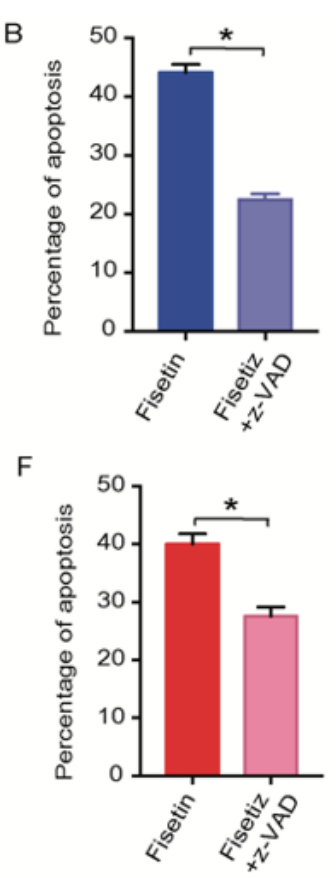
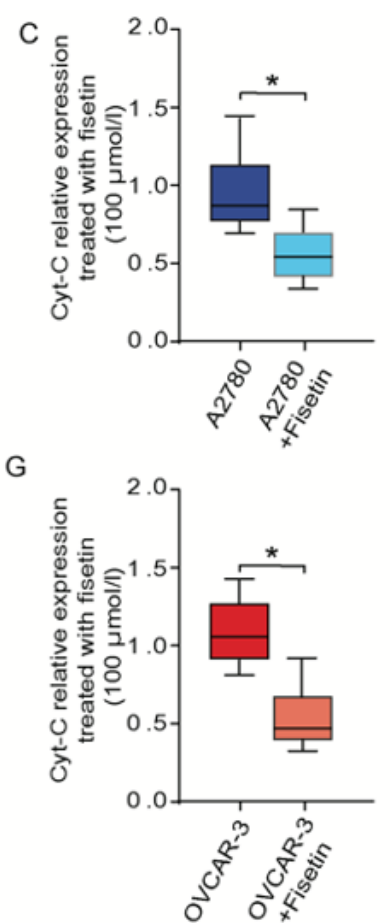
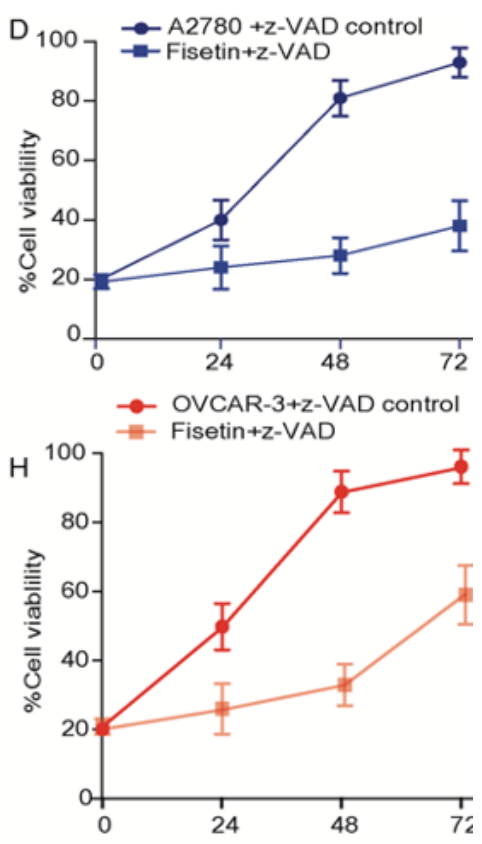

Figure 2

Mechanism other than cell apoptosis involved in OC cell death induced by fisetin. (A) Cytometric results of Annexin V and Propidium iodide (AV/PI) staining with Ho8910 cells. (B)Quantification of apoptotic Ho8910 cells treated by fisetin $\pm z-V A D$. (C) Relative expression level of Cyt-C translated in the mitochondria of Ho8910 cells treated with or without fisetin $(100 \mathrm{mmol} / \mathrm{L})$ for $72 \mathrm{~h}$. (D) Comparison of fisetin treated Ho8910 cell growth in a time-dependent manner, revealing the inhibition on apoptosis by zVAD failed to promote cell growth after fisetin treatment. (E-H) Identical experiments and intervention were administrated on the other $\mathrm{OC}$ cell line, A2780 cells. ${ }^{*}, \mathrm{p}<0.05$. 


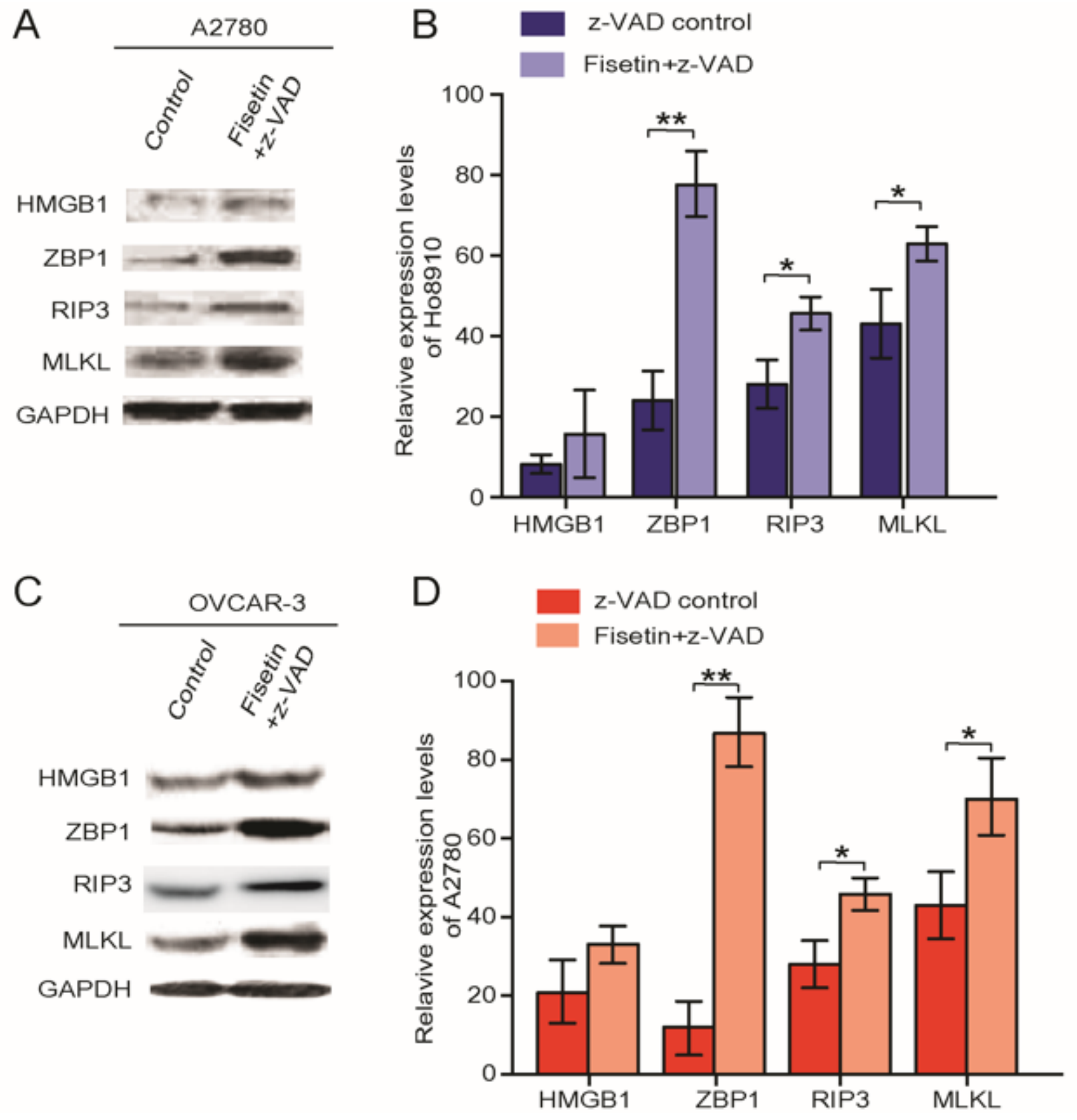

Figure 3

Cell necroptotic pathways regulates fisetin induced cell death. (A) On Ho8910 cells, the expression of HMGB1, ZBP1, RIP3 and MLKL in the control and fisetin + z-VAD treatment group by western blot. (B) Quantification and comparison on the level of HMGB1, ZBP1, RIP3 and MLKL expression. (C, D) Western blot results and quantification on HMGB1, ZBP1, RIP3 and MLKL in A2780 cells under respective intervention. *, $p<0.05$. 


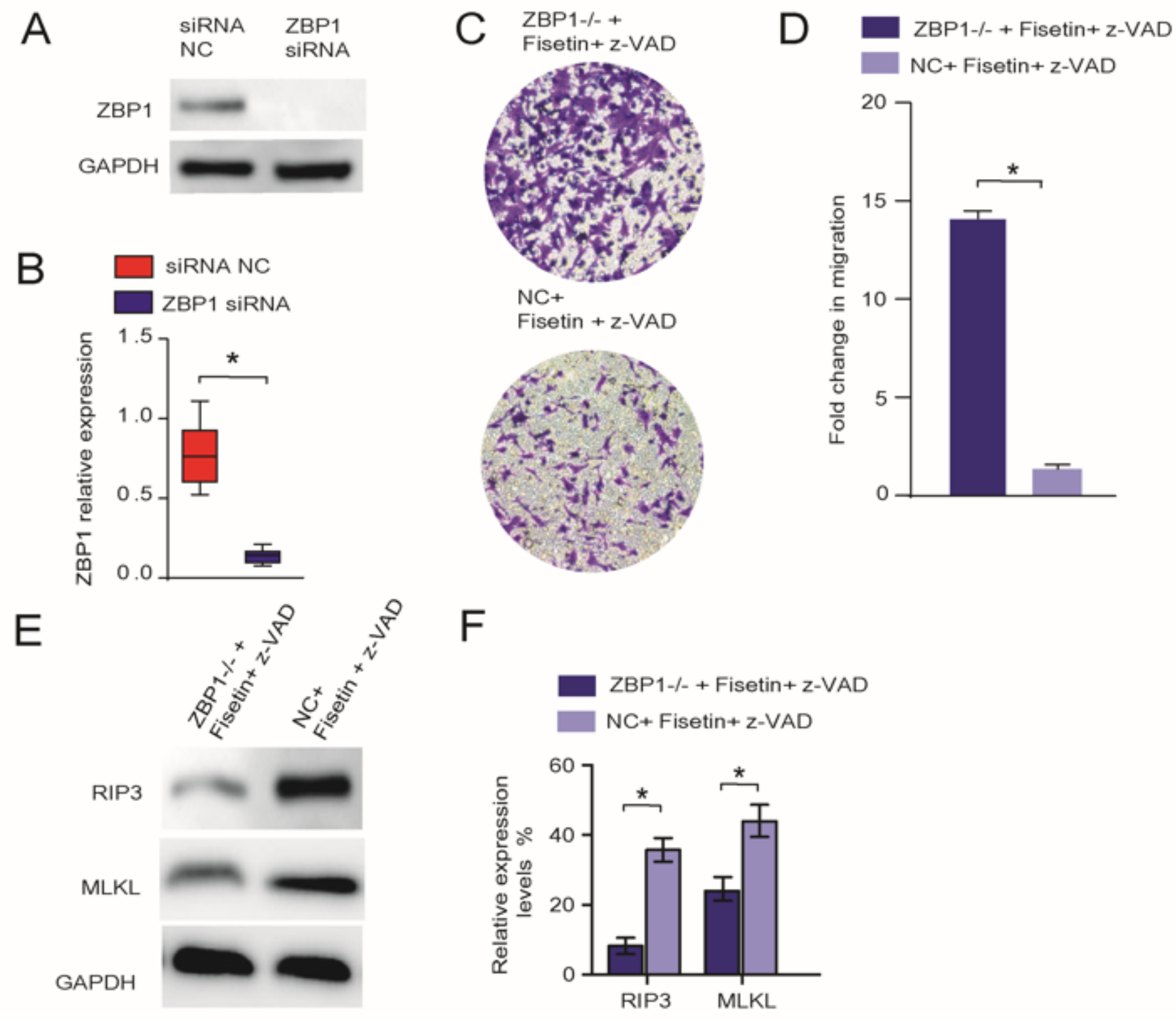

Figure 4

ZBP1 knockdown increased cell migration of Ho8910 cells blocking fisetin induced apoptosis. (A) Western blotting on both ZBP1 knockdown (ZBP1-/-) group and control group in which Ho8910 cells were treated by fisetin + Z-VAD. (B) Quantification of ZBP1 expression with or without ZBP siRNA administration. (C) In vitro invasion assay between respective groups. (D) MTT assay indicated difference of cell migration between indicated groups. (E) Western blotting and (F) quantitative expression level targeting RIP3 and MLKL in both ZBP1-/- and normal Ho8910 cells, both of which were treated by fisetin $+z-V A D . *, p<0.05$. 
A

$$
\begin{array}{ll}
\text { SiRNA } & \text { ZBP1 } \\
\text { NC } & \text { siRNA }
\end{array}
$$

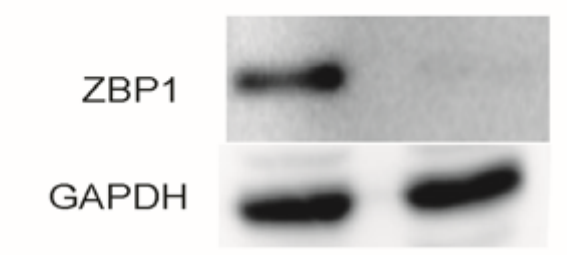

B
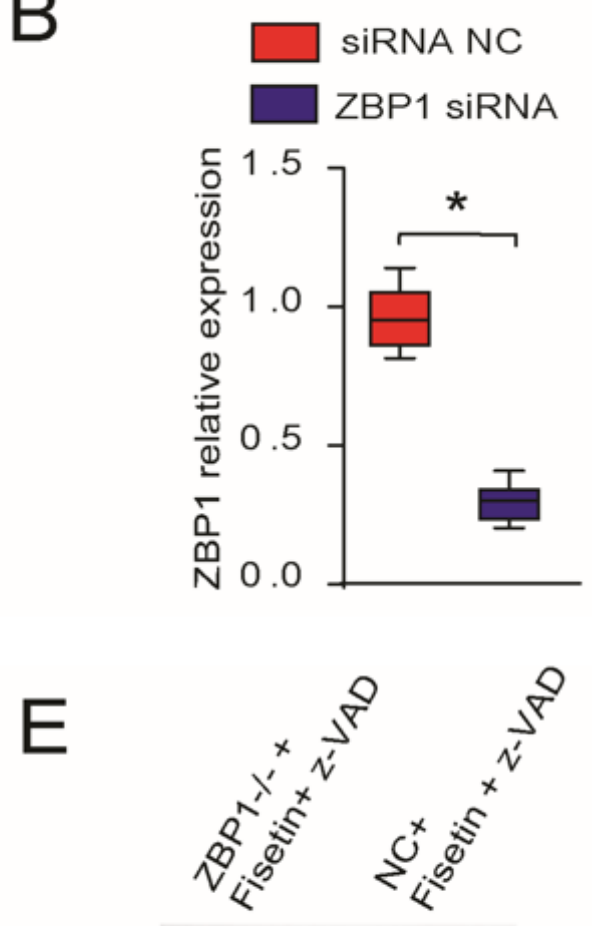

RIP3

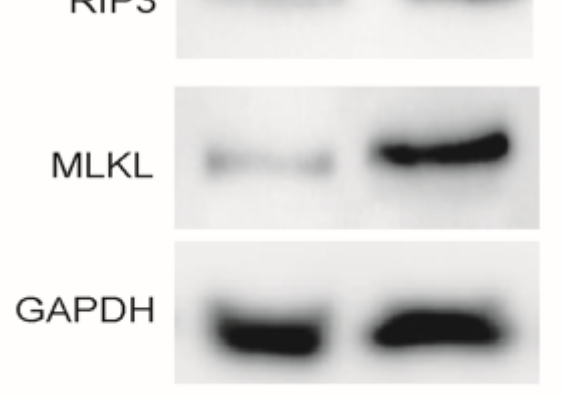

C

ZBP1-/- +

Fisetin + z-VAD

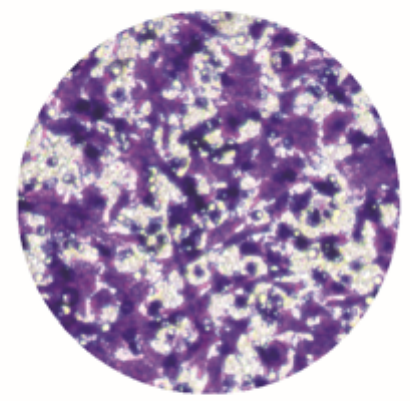

$\mathrm{NC}+$

Fisetin + z-VAD

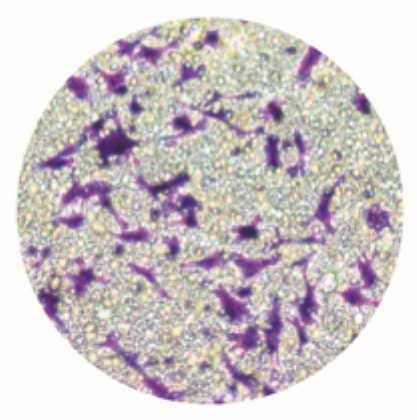

D

ZBP1-/- + Fisetin + z-VAD $\mathrm{NC}+$ Fisetin+ $\mathrm{z}-\mathrm{VAD}$

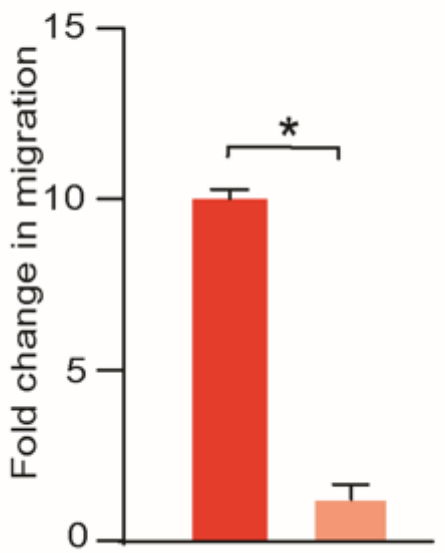<smiles>F</smiles>

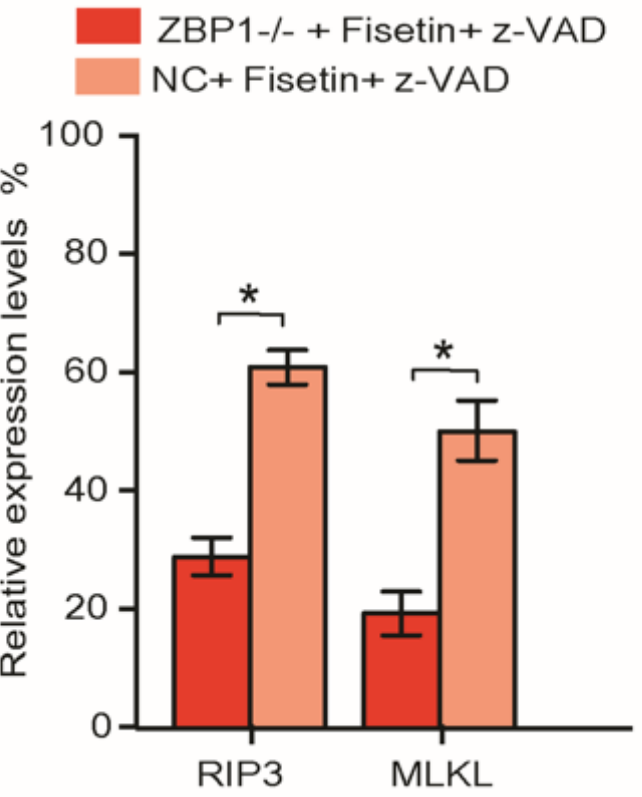

\section{Figure 5}

ZBP1 knockdown increased cell migration of A2780 cells blocking fisetin induced apoptosis. (A) Western blotting on both ZBP1 knockdown (ZBP1-/-) group and control group in which A2780 cells were treated by fisetin + Z-VAD. (B) Quantification of ZBP1 expression with or without ZBP siRNA administration. (C) In vitro invasion assay between respective groups. (D) MTT assay indicated difference of cell migration 
between indicated groups. (E) Western blotting and (F) quantitative expression level targeting RIP3 and MLKL in both ZBP1-/- and normal A2780 cells, both of which were treated by fisetin + z-VAD. *, $p<0.05$.

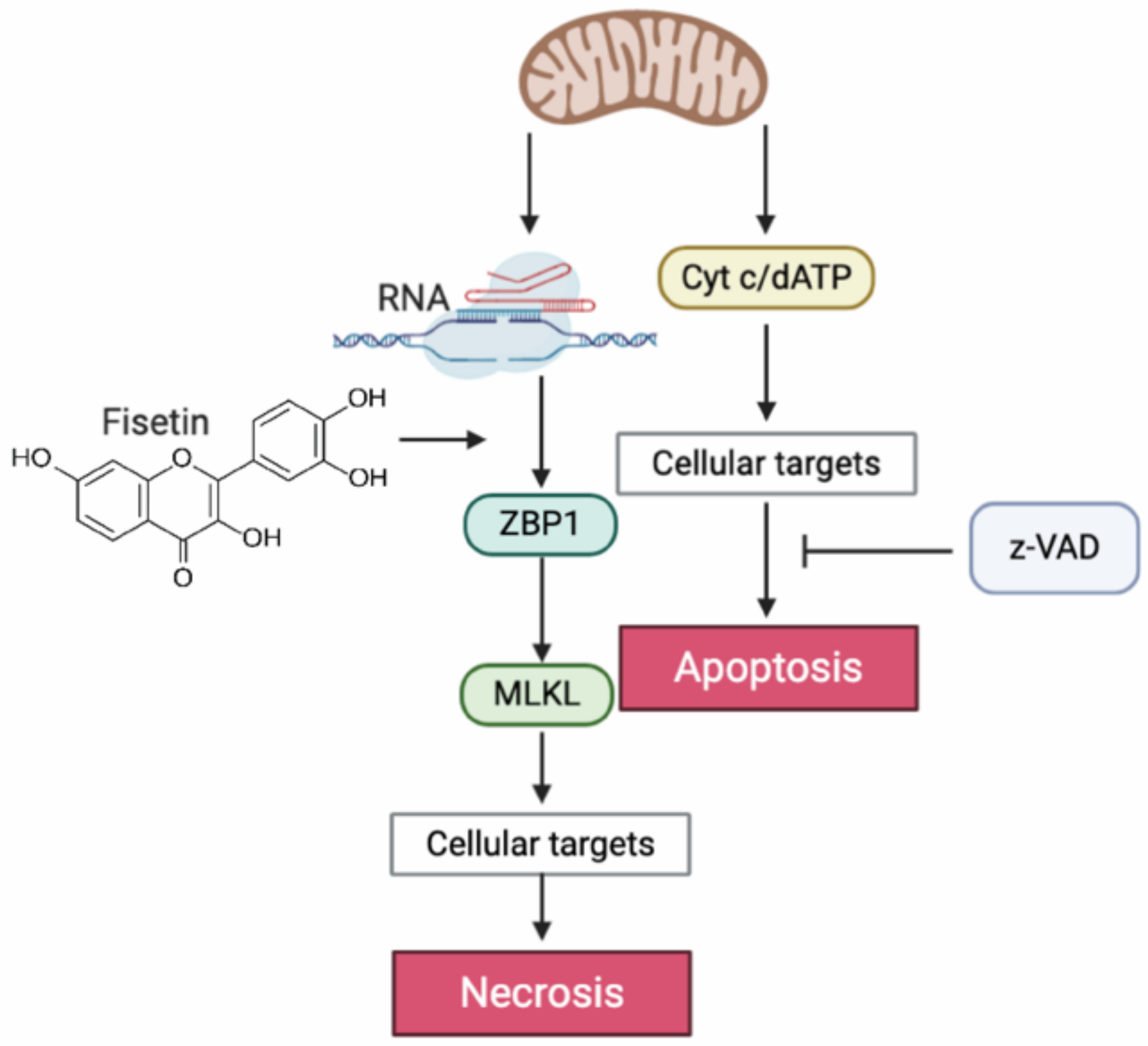

Figure 6

Schematic illustration on the mechanism related to $\mathrm{OC}$ cell death induced by fisetin. 